\title{
AN EXTENSION OF THE CHEN-BEURLING-HELSON-LOWDENSLAGER THEOREM
}

\author{
HAIHUI FAN, Don HADWIN AND WENJING LIU
}

Abstract. Yanni Chen [3] extended the classical Beurling-Helson-Lowdenslager theorem for Hardy spaces on the unit circle $\mathbb{T}$ defined in terms of continuous gauge norms on $L^{\infty}$ that dominate $\|\cdot\|_{1}$. We extend Chen's result to a much larger class of continuous gauge norms. A key ingredient is our result that if $\alpha$ is a continuous normalized gauge norm on $L^{\infty}$, then there is a probability measure $\lambda$, mutually absolutely continuous with respect to Lebesgue measure on $\mathbb{T}$, such that $\alpha \geqslant c\|\cdot\|_{1, \lambda}$ for some $0<c \leqslant 1$.

Mathematics subject classification (2010): Primary: 46E20, 30H10, Secondary: 30J99, 47L10. Keywords and phrases: Gauge norm, Hardy space, Beurling theorem.

\section{REFERENCES}

[1] A. Beurling, On two problems concerning linear transformations in Hilbert space, Acta Math. 81 (1949) 239-255.

[2] Y. CHEN, Lebesgue and Hardy spaces for symmetric norms I, arXiv: 1407.7920 [math. OA] (2014).

[3] Y. CHEN, A general Beurling-Helson-Lowdenslager theorem on the disk, Advances in Applied Mathematics 87 (2017) 1-15.

[4] P. DuREN, Theory of $H^{p}$ spaces, Academic Press, New York, 1970.

[5] P. Halmos, Shifts on Hilbert spaces, J. Reine Angew. Math. 208 (1961) 102-112.

[6] H. Helson, Lectures on Invariant Subspace, Academic Press, New York-London, 1964.

[7] H. Helson and D. Lowdenslager, Prediction theory and Fourier series in several variables, Acta Math. 99 (1958) 519-540.

[8] K. Hoffman, Analytic functions and logmodular Banach algebras, Acta Math. 108 (1962) 271-317.

[9] N. K. NikOLSKII, Treatise on the shift operator, Spectral function theory, With an appendix by S. V. Hruscev and V. V. Peller, Translated from the Russian by Jaak Peetre, Grundlehren der Mathematischen Wissenschaften, 273. Springer-Verlag, Berlin, 1986.

[10] T. P. SRIniVASAn, Simply invariant subspaces, Bull. Amer. Math. Soc. 69 (1963) 706-709. 